\title{
Facile fabrication of stretchable Ag nanowire/polyurethane electrodes using high intensity pulsed light
}

\author{
Yang Yang ${ }^{1}$, Su Ding ${ }^{2,3}$, Teppei Araki ${ }^{2}(\varangle)$, Jinting Jiu $^{2}$, Tohru Sugahara ${ }^{2}$, Jun Wang ${ }^{2,3}$, Jan Vanfleteren ${ }^{1}(\varangle)$,
} Tsuyoshi Sekitani ${ }^{2}$, and Katsuaki Suganuma ${ }^{2}$

${ }^{1}$ Center for Microsystems Technology, IMEC and Ghent University, 9052 Gent-Zwijnaarde, Belgium

${ }^{2}$ The Institute of Scientific and Industrial Research, Osaka University, Ibaraki, Osaka 567-0047, Japan

${ }^{3}$ State Key Laboratory of Advanced Welding and Joining, Harbin Institute of Technology, Harbin 150001, China

Received: 21 July 2015

Revised: 14 September 2015

Accepted: 13 October 2015

(C) Tsinghua University Press and Springer-Verlag Berlin Heidelberg 2015

\section{KEYWORDS}

silver nanowires, stretchable electrode, photonic sintering, nanofabrication

\begin{abstract}
Silver nanowires (AgNWs) have emerged as a promising nanomaterial for next generation stretchable electronics. However, until now, the fabrication of AgNWbased components has been hampered by complex and time-consuming steps. Here, we introduce a facile, fast, and one-step methodology for the fabrication of highly conductive and stretchable AgNW/polyurethane (PU) composite electrodes based on a high-intensity pulsed light (HIPL) technique. HIPL simultaneously improved wire-wire junction conductivity and wire-substrate adhesion at room temperature and in air within $50 \mu \mathrm{s}$, omitting the complex transfer-curing-implanting process. Owing to the localized deformation of PU at interfaces with AgNWs, embedding of the nanowires was rapidly carried out without substantial substrate damage. The resulting electrode retained a low sheet resistance (high electrical conductivity) of $<10 \Omega$ /sq even under $100 \%$ strain, or after 1,000 continuous stretching-relaxation cycles, with a peak strain of $60 \%$. The fabricated electrode has found immediate application as a sensor for motion detection. Furthermore, based on our electrode, a light emitting diode (LED) driven by integrated stretchable AgNW conductors has been fabricated. In conclusion, our present fabrication approach is fast, simple, scalable, and costefficient, making it a good candidate for a future roll-to-roll process.
\end{abstract}

\section{Introduction}

Recent advancements in stretchable electronics have opened up new opportunities for a wide range of applications. Numerous efforts have been made to convert electronic systems that are traditionally constrained to rigid and flat formats into the next generation, that will be bendable, compressible, stretchable, twistable, conformable, or deformable to arbitrary 3D shapes. Examples of this work can be

Address correspondence to Jan Vanfleteren, jan.vanfleteren@elis.ugent.be; Teppei Araki, araki@sanken.osaka-u.ac.jp 
found in areas including large area electronics, wearable devices, sensors, light emitting diodes (LEDs), and batteries [1-17]. To accommodate large mechanical deformations during stretching while maintaining the electrical performance and stability of the system, either the materials themselves or the structures need to be stretchable [1]. The traditional approach to this problem has been to engineer non-stretchable conductors into novel shapes, followed by encapsulation in an elastic material to achieve stretchable performance, which requires optimized conductor design and precise patterning [1-8].

However, the current trend is to assemble stretchable nanomaterials into elastomeric substrates which then act as both electrical and stretchable materials, taking full advantage of nanomaterials that are intrinsically stretchable at the macroscopic scale. For example, graphene, carbon nanotubes, and metal nanowires have recently attracted much attention in this field [12-24]. Carbon nanomaterials have been the dominant research topic in the field of stretchable conductors. However, they suffer from poor electrical conductivity [25]. Silver nanowires (AgNWs) have higher conductivity than carbon nanomaterials or any other metal nanowires. Their conductivity is at least one order of magnitude higher than single-walled nanotubes (SWNTs) and three orders of magnitude higher than graphene [25]. In addition, AgNWs demonstrate very good mechanical flexibility [26]. $\mathrm{Xu}$ and $\mathrm{Zhu}$ reported a stretchable electrode with AgNWs embedded in the surface layer of polydimethylsiloxane (PDMS) prepared by a $12 \mathrm{~h}$ curing cycle of liquid PDMS at $65^{\circ} \mathrm{C}$ [27]. Lee et al. fabricated a AgNW-based stretchable electrode by transferring the AgNWs onto a pre-strained elastomeric substrate [28]. The substrate was then annealed for $2 \mathrm{~h}$ at $220^{\circ} \mathrm{C}$ in a convection oven. Hu et al. fabricated a AgNW electrode by embedding the AgNWs in the surface layer of an elastomeric polyurethane matrix [29]. The fabrication process involved annealing the drop-casted AgNWs on a glass plate for $30 \mathrm{~min}$ at $190{ }^{\circ} \mathrm{C}$, followed by drop-casting the urethane compounds onto the AgNWs and curing for $24 \mathrm{~h}$. Liang et al. fabricated a AgNW/poly(urethane acrylate) (PUA) composite electrode by coating the AgNWs with PUA precursor solution, followed by curing the coated material [30]. As can be seen, these works require rather complex and time-consuming fabrication steps in order to encapsulate or embed the AgNWs in the matrix to achieve a composite structure. Moreover, the high temperature sintering required in these processes hinders the use of temperature-sensitive materials. Fortunately, a simpler and much faster technique called high-speed photonic sintering has paved the way to directly embed AgNWs in a matrix at room temperature [31-33]. Photonic sintering has been recently developed to replace conventional thermal sintering of metal nanoparticles, and works rapidly at room temperature on large scales through lightinduced heat generation in metallic nanostructures [31-37]. Jiu et al. also found that the residual heat generated by photonic sintering in the metal network could be used to achieve strong adhesion between the network and a flexible substrate due to the deformation of the polymer substrates [31,32]. Provided that most of the stretchable devices are fabricated based on a polymer matrix, which can easily be deformed with heat, we believe photonic sintering is a potential candidate for the creation of a composite structure with stretchable substrates and metallic nanowires in a much simpler and faster manner.

Herein, we report the facile fabrication of a stretchable AgNW/polyurethane (PU) electrode based on the high-intensity pulsed light (HIPL) technique. This technique employs high intensity light to generate extreme heat localized specifically on the metallic nanostructure [33-35]. Owing to the strong dependence of the plasmonic light concentration on the distance between the nanowires, nano-welding between nanowires can be accomplished, resulting in high electrical conductivity. The residual heat will also embed the network into the soft polymer substrate, while being restricted to the AgNW-PU interface, preventing substantial damage to the substrate. The mechanism of the proposed fabrication methodology has been further studied via simulations. The performance of the resulting electrodes has been evaluated extensively under various conditions. Moreover, the effect of NW length on the performance of the resulting electrodes has been investigated. To demonstrate the potential applications of our electrodes, we have utilized them in several stretchable devices including a stretchable LED and a wearable sensor for human motion detection. 


\section{Experimental}

\subsection{Materials}

AgNWs of two lengths were used in this work, which were synthesized according to a previously reported method at $110{ }^{\circ} \mathrm{C}$ for $12 \mathrm{~h}$ for long NWs, and $150{ }^{\circ} \mathrm{C}$ for $2 \mathrm{~h}$ for short NWs [38]. The long NWs measured 30-60 $\mu \mathrm{m}$ in length and $85 \mathrm{~nm}$ in diameter, and the short NWs were 10-20 $\mu \mathrm{m}$ in length and $70 \mathrm{~nm}$ in diameter. Unless otherwise mentioned, all the results presented here were obtained using the long NWs. The PU sheet was obtained from Takeda Sangyo Co., Ltd. Silver paste (D-550), used to fabricate the conformal electrodes, was purchased from Fujikura Kasei Co., Ltd.

\subsection{Fabrication of AgNW/PU electrodes}

The fabrication steps of the composite electrode are illustrated in Fig. 1. Firstly, the PU was cleaned gently with ethanol and placed on a flat surface. Then a dispersion of AgNWs in ethanol ( 2.5 wt.\%) was spray-deposited onto the PU substrate. The distance between the substrate and the airbrush (PEACE 3, Airtex, Japan) was about $10 \mathrm{~cm}$. After drying for a few seconds in air, the AgNW films were treated with HIPL (PulseForge 3300, Novacentrix, Austin, TX, USA) at various light intensities with exposure time fixed to $50 \mu$ s at room temperature and in air. This resulted in composite electrodes featuring a AgNW film inlaid on the surface of PU. For stretchability tests, silver paste was printed on both ends of the resulting AgNW films and dried for $2 \mathrm{~min}$ at room temperature. The effective area of the AgNW electrode was $12 \mathrm{~mm} \times$ $15 \mathrm{~mm}$.

(a)

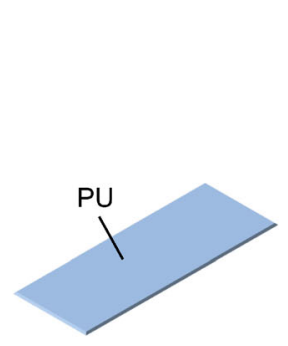

(b)

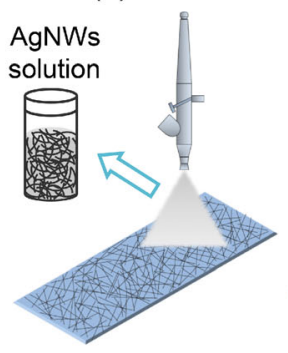

\subsection{Characterization of the electrode}

Optical transmittance spectra over the wavelength range of 300-800 nm were measured by a UV-visible near infrared spectrophotometer (V670, JASCO Corp.) using a sheet of pure PU as a reference. The sheet resistance (SR) of the electrodes was measured using the four-probe method with a surface resistivity meter (Loresta GP T610, Mitsubishi Chemical Analytech Co., Ltd.). SEM images of the samples were recorded by a FE-SEM (SU8020, Hitachi, Japan). A tensile testing machine (EZ test, Shimadzu, Japan) was applied to stretch the electrodes from 12 to $24 \mathrm{~mm}$ (100\% strain) and release them back to $12 \mathrm{~mm}$ uniaxially at speeds of $2,4,60,120$, and $480 \mathrm{~mm} / \mathrm{min}$. The electrical resistance of the samples during tensile stretching was measured with an Agilent Technologies 34410A multimeter and an Agilent Technologies 11059A Kelvin probe set (Agilent Technologies, Santa Clara, USA) through the four-point probe method. Furthermore, continuous stretching-relaxation tests over 1,000 cycles were carried out using samples with initial SRs of 2.5, 1.1, and $0.6 \Omega / \mathrm{sq}$, or with peak strains of $20 \%, 40 \%$, and $60 \%$, respectively.

\subsection{Fabrication of LED integrated conductor}

An island was embedded in the PU substrate by hot pressing. A square of paper $(5 \mathrm{~mm} \times 5 \mathrm{~mm})$ was cut out from a membrane filter (cellulose acetate, Advantech) and sandwiched between two $2 \mathrm{~cm} \times 4 \mathrm{~cm}$ PU films. In order to bond the PU sheets to each other and to the paper island, the specimen was pressed at a pressure of $6 \mathrm{MPa}$ and a temperature of $130{ }^{\circ} \mathrm{C}$ for $2 \mathrm{~min}$. Long AgNWs were spray-coated on the surface of the PU, then treated with HIPL to achieve a SR of $6 \Omega /$ sq.

(c)

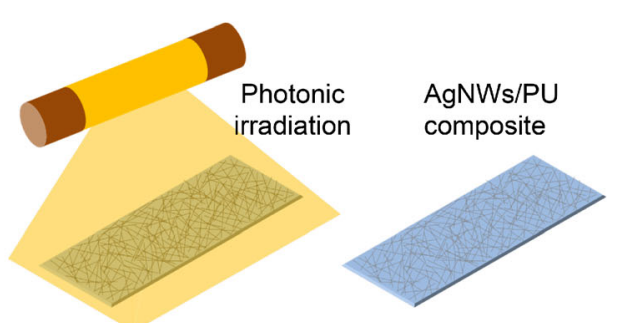

Figure 1 Schematic representation of the fabrication steps of AgNW/PU electrodes. (a) PU gently cleaned and placed flat. (b) Spray deposition of AgNWs onto PU. (c) Photonic sintering over the whole area. (d) Resulting AgNW/PU composite electrode. 
Areas of the AgNW network were isolated using a green laser (we used a laser having a wavelength of $532 \mathrm{~nm}$ and a pulsed duration of nanosecond level) to fabricate electrically separated contact pads to act as the anode and cathode of the LED. A red LED chip (PICOLED, low current type, Rohm) was mounted above the center of the island by connecting the AgNW electrodes to the LED contacts with a conductive adhesive diluted with a mixture of water and ethanol [22].

\section{Results and discussion}

The SEM images of the as-deposited AgNW percolation network on PU (i.e., after the step depicted in Fig. 1(b)) are shown in Figs. 2(a) and 2(b). The nanowires were found to be randomly distributed on the PU surface, forming a network structure whose junctions led to the conductivity of the electrode. The formation of these junctions was mainly driven by pressure from the airbrush, gravity, van der Waals' interactions between the nanowires, and capillary forces due to evaporation of solvent during electrode fabrication [32]. The close-up SEM image shown in Fig. 2(b) clearly shows that these AgNWs are randomly stacked and loosely coated on the surface of the PU, and that no embedding of AgNWs is observed. Moreover, polyvinylpyrrolidone (PVP) residuals (used in the synthesis of AgNWs) seem to remain on the surface of nanowires, and are clearly visible at some junctions. Notably, with a single exposure of high intensity light for $50 \mu \mathrm{s}$, the clear wire-wire and wire-PU interfaces immediately disappeared (Figs. 2(c) and 2(d)). The randomly stacked network became tightly connected and was buried in the PU substrate, giving rise to a strong physical adhesion to the PU substrate. The nanowires at the top of the stack are fully or partially embedded into the PU substrate. As shown in Fig. 2(e),
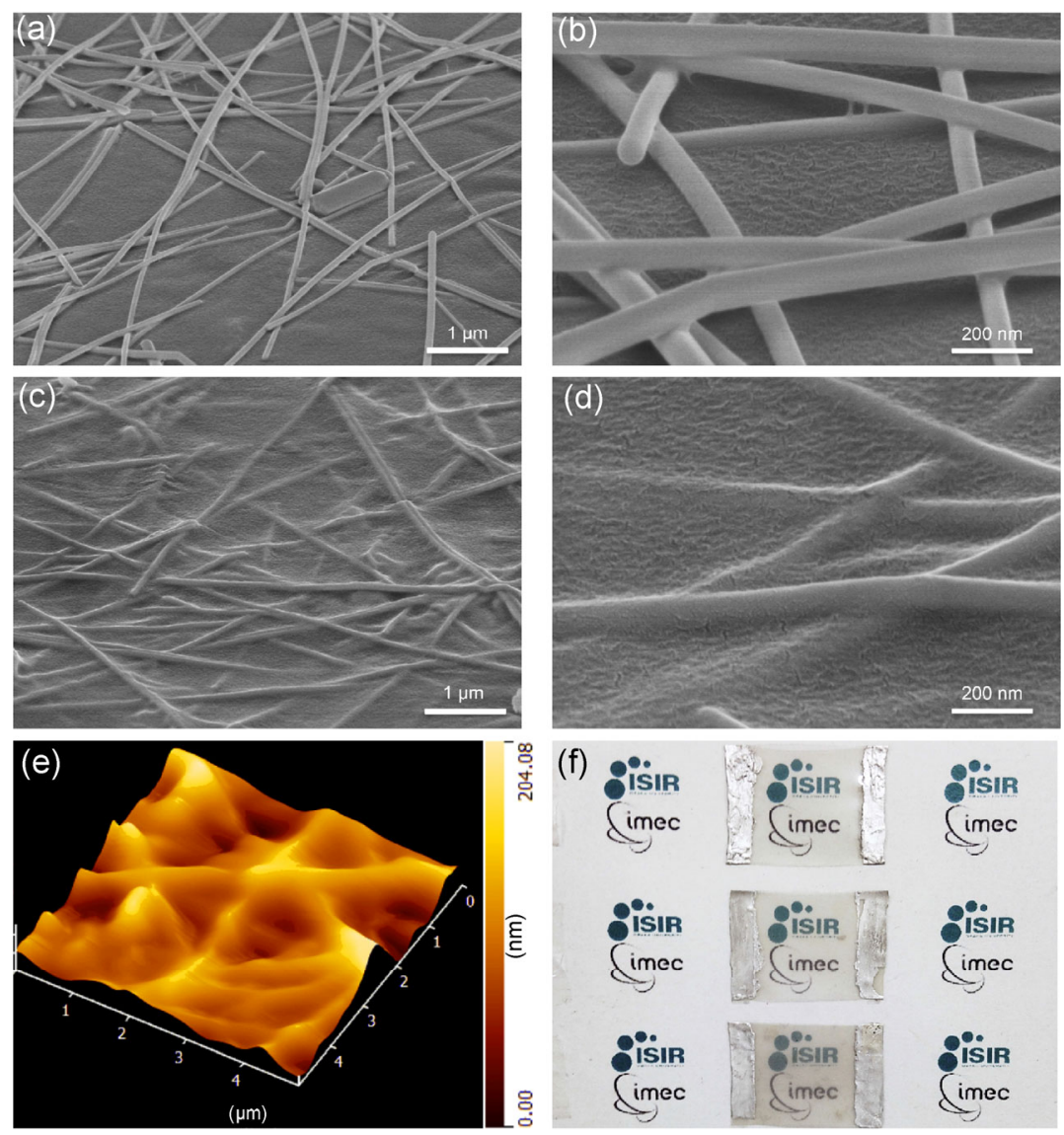

Figure 2 (a) and (b) Tilted SEM images of as-prepared AgNWs deposited on PU substrate before HIPL. (c) and (d) Tilted SEM images and (e) AFM image of AgNW/PU electrodes after HIPL. (f) Digital photograph of AgNW/PU electrode after HIPL, transmittance is $80 \%$, $60 \%$, and $40 \%$ from top to bottom.

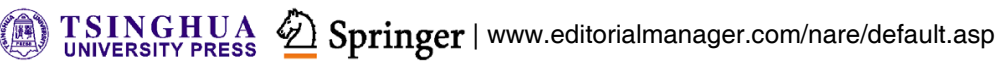


surface roughness measured by atomic force microscopy (AFM) was $204 \mathrm{~nm}$, which is far smaller than the original roughness of over $500 \mathrm{~nm}$, suggesting that the majority of the network was buried in the PU substrate. Figure 2(f) is a digital photograph of electrodes fabricated with specific AgNW loadings. Their transmittances are $80 \%, 60 \%$, and $40 \%$ (from top to bottom).

Several issues must be addressed to allow largescale fabrication and broad application of AgNW-based stretchable electrodes (as demonstrated by Figs. 2(a) and 2(b)). One challenge for simple and effective assembly of nanowire networks is the removal of the insulating PVP surfactant coating from the NWs after deposition to ensure conductivity through wire-wire junctions. This is usually done through treatments such as thermal annealing [39-42] or mechanical pressing $[39,40,43]$. However, thermal annealing (often over $150{ }^{\circ} \mathrm{C}$ ) is very time consuming (from minutes to hours) and excludes the use of many heat-sensitive materials, while pressing techniques can cause damage to delicate substrates or devices. Another challenge in the implementation of a nanowire network is obtaining strong adhesion between the network and the substrate for stable and robust performance. To successfully improve the adhesion, substrate surface modification has been used [40,41,44], a strong conformal pressure has been applied [40,43], and in-situ polymerization [45, 46] and surface encapsulation have been reported [26, 27, $41,42,47]$. However, these processes are complex and time-consuming, in addition to the fact that they may change the properties of the substrate materials. For practical usage, the process of forming the AgNW films should be simple and cost-effective, while maintaining the performance of the materials. The HIPL technique meets these criteria.

Figure 3 shows the differences between the conventional process and our current fabrication technique. For the conventional process (Fig. 3(a)), AgNWs are first deposited on a heat-resistant temporary carrier (usually glass or silicon), followed by thermal annealing of the complete stack at an elevated temperature (often higher than $150{ }^{\circ} \mathrm{C}$ ). This step usually takes between minutes and hours depending on the sintering temperature. After this, liquid elastomer is poured onto the stack, followed by a curing process. Again, this step

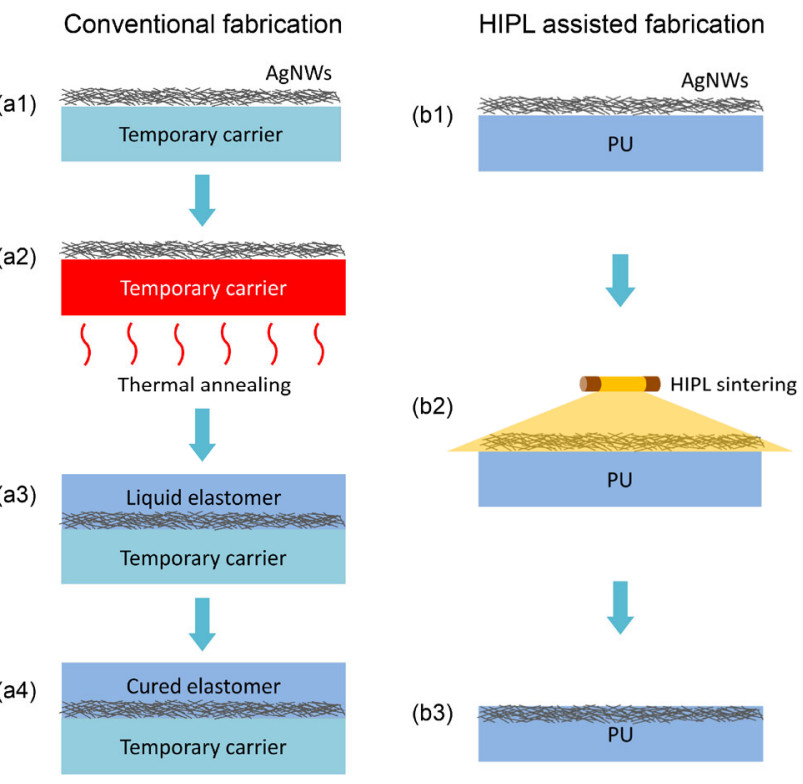

Figure 3 A cross-sectional view showing the comparison of HIPL-assisted vs. conventional fabrication process. HIPL sintering simultaneously improves wire-wire connectivity and wire-substrate adhesion at a single step. The complete process takes only $50 \mu \mathrm{s}$. On the other hand, conventional methods address these two issues over multiple steps, for instance with a thermal annealing to improve wire-wire connectivity, followed by an in-situ polymerization from liquid elastomer for wire-substrate adhesion.

is time consuming, and the time needed for the curing depends on the chemistry of the components.

One of the advantages of HIPL assisted fabrication processes over conventional techniques is that they can address these two issues simultaneously with a single light exposure, as shown in Fig. 3(b). The samples were fixed $10 \mathrm{~cm}$ from the lamp and sintered with different intensities of light at room temperature and in air. The xenon lamp used has a wide emission spectrum covering the range $100-1,000 \mathrm{~nm}$ and has an effective area of $7.5 \mathrm{~cm} \times 15 \mathrm{~cm}$. The light intensity can be controlled by varying the voltage and pulse duration, allowing tuning from several $\mathrm{mJ} / \mathrm{cm}^{2}$ to several hundreds of $\mathrm{mJ} / \mathrm{cm}^{2}$. In contrast to heat- or pressure-based sintering processes that involve continuous treatment of the whole sample for a long period, HIPL generates extreme heat only on the metallic nanostructure for an extremely short period (only tens of microseconds) [33-35]. This transient process allows the use of a higher temperature, hence dramatically shortens the sintering process. As a result, wire-wire junction conductivity was rapidly improved, 
resulting in high conductivity. After HIPL, SR of the electrode decreased from 10.9 to $1.8 \Omega / \mathrm{sq}$, with a transmittance of $50 \%$. When the same electrode was heated at $100{ }^{\circ} \mathrm{C}$ for $20 \mathrm{~min}$, the SR decreased from 10.9 to $5.8 \Omega /$ sq.

When the AgNW electrode is exposed to high intensity light, it absorbs a large amount of light at 350-450 nm within several microseconds [48], which is then either converted to thermal energy, or induces the diffusion and assembly of Ag atoms on the surface of NWs to sinter the AgNWs, producing high conductivity. Hence, the Ag loading and the parameters of the light are important for the improvement of conductivity of the AgNW/PU electrodes. The temperatures and their evolution at different parts of the electrode have been simulated (more details on the simulation are available in Table S1 and Fig. S1 in the Electronic Supplementary Material (ESM)), and more results on the influence of the pulsed light irradiation are available in Fig. S2 in the ESM. According to the simulation, the temperature at the top of AgNW film and at the interface between the AgNW film and the PU was almost the same. This is probably due to the very high thermal conductivity of $\mathrm{Ag}$ and the small thickness of the AgNWs. The high thermal conductivity of Ag allows the extreme heat to be rapidly spread across the whole network, leading to an increase of its temperature. On the other hand, the temperature at the bottom of the PU was almost unchanged $\left(25^{\circ} \mathrm{C}\right)$ due to the very poor thermal conductivity of PU. As a result, the diffusion of the heat to the bottom of the PU was hindered, preserving the heat in the AgNWs. After an extremely short amount of time, the temperature rise $\left(>700{ }^{\circ} \mathrm{C}\right)$ led to the softening or melting of adjacent PU (which has a melting point of around $150{ }^{\circ} \mathrm{C}$ ). The soft structure easily engulfed the heavy $\mathrm{Ag}$, resulting in the embedding of the AgNW network into the PU. As the light is transiently stopped and PU has a low thermal conductivity $(0.03 \mathrm{~W} / \mathrm{mK})$, the PU substrate was only locally damaged at the interface with the AgNWs. According to the simulation, the estimated depth of softened PU was between 5 and $10 \mu \mathrm{m}$. The degradation of the elastic properties of PU caused by this damage will inevitably affect the performance of AgNW/PU electrodes. Compared to undamaged PU, the deteriorated PU showed poorer stretching- relaxation properties. It should be mentioned that the software estimated the temperature by assuming a dense Ag film on top of the substrate. In the actual experiment, the film exists as a percolating network structure with voids and gaps (and therefore much less $\mathrm{Ag}$ ), meaning the simulation temperatures were much higher than those seen in reality. Hence, the actual depth of softened PU was far lower than $10 \mu \mathrm{m}$. However, the instantaneous heat is a weakness of the HIPL technique. As we have mentioned, the HIPL technique is fast, simple, and large-scale. If HIPL parameters such as light intensity, exposure time, and pulse number are carefully selected, the damage of the substrate can be minimized. This embedding process greatly enhanced the adhesion between the AgNWs and the PU substrate. The strong adhesion between AgNWs and PU substrate achieved by HIPL was confirmed by a stretchability test, as illustrated in Fig. S3 in the ESM. The HIPL-treated AgNW electrode endured many stretching-relaxation cycles without significant changes in resistance, and recovered after relaxation. In contrast, the as-prepared sample shows rapid increase of resistance upon each cycle, and lost its conductivity completely after $100 \mathrm{~s}$.

To investigate the influence of the electrode's initial SR on its stretchability, electrodes with different initial SRs were stretched to $100 \%$ strain at a stretching speed of $2 \mathrm{~mm} / \mathrm{min}$ at room temperature. As shown in Fig. 4(a), the electrode with an initial SR of $2.3 \Omega / s q$ (initial resistance $=3.4 \Omega$ ) could be stretched by up to $80 \%$, with resistance increasing by more than three orders of magnitude. However, on further stretching, the electrode resistance steeply increased to infinity. For an electrode with a lower initial SR of $1.1 \Omega / \mathrm{sq}$ (initial resistance $=1.9 \Omega$ ), the maximum allowable strain increased to $100 \%$, and the increase in resistance was only slightly more than one order of magnitude. For an electrode of an even lower initial SR of $0.6 \Omega / \mathrm{sq}$ (initial resistance $=1.2 \Omega$ ), the increase in resistance was slightly lowered to around one order of magnitude. Electrodes obtained by our simple process feature both very low SR and high stretchability. Our results outperform, or at least are comparable to, previously reported results with similar transmittance that use much more complex fabrication methods [41,44]. Figure S4 in the ESM shows that the increased 

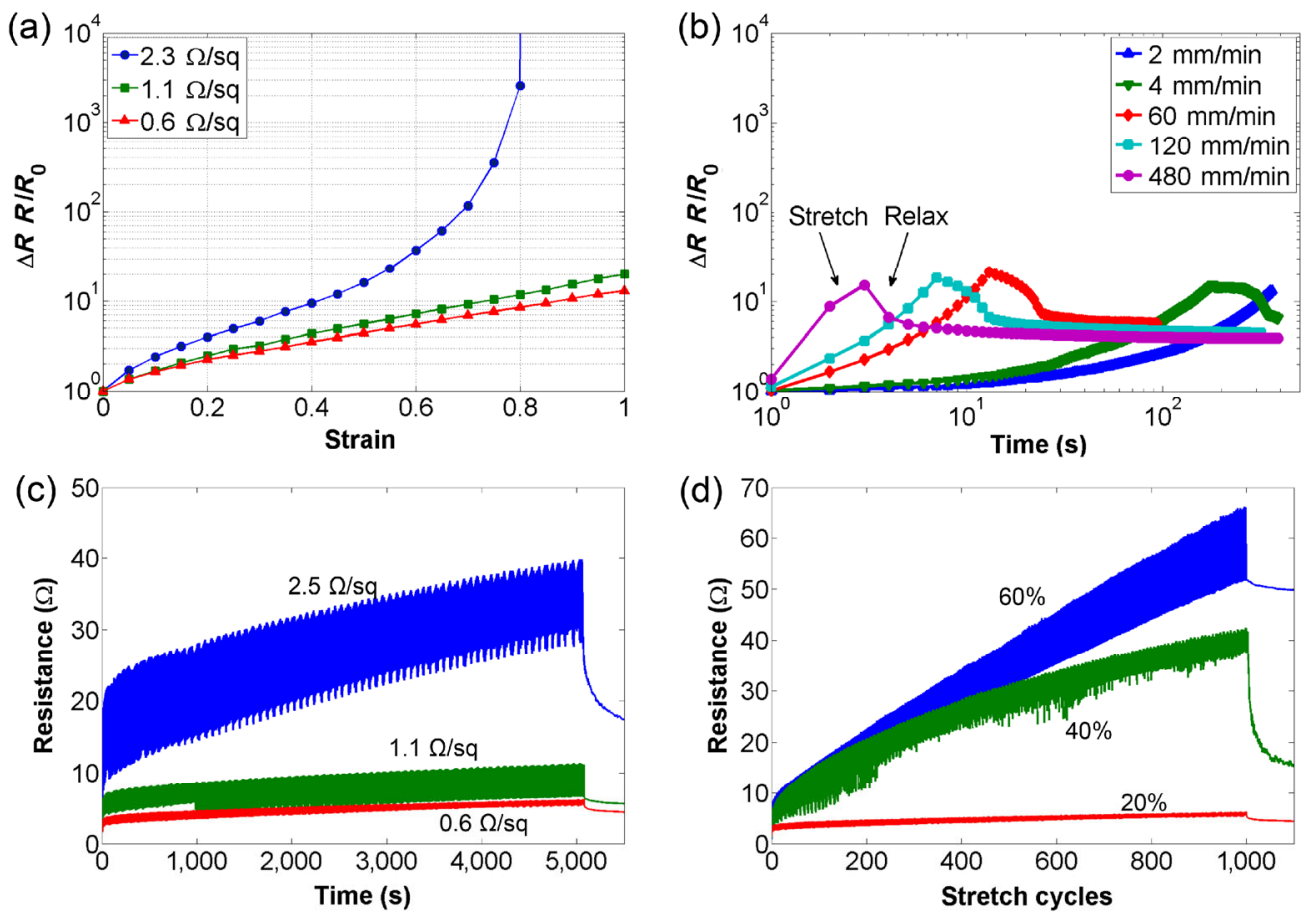

Figure 4 Capability of as-prepared stretchable electrodes. (a) Resistance change with strain up to $100 \%$ with various initial SRs at a stretching speed of $2 \mathrm{~mm} / \mathrm{min}$. (b) Resistance change with strain up to $100 \%$ at different stretching speeds with an initial SR of $0.6 \Omega /$ sq. (c) Transient resistance measured over 1,000 cycles of stretching-relaxation between 0 and 20\% strain with original SRs of 2.5, 1.1, and $0.6 \Omega / \mathrm{sq}$ at a stretching speed of $60 \mathrm{~mm} / \mathrm{min}$. (d) Transient resistance measured over 1,000 cycles of stretching-relaxation between 0 and $20 \% / 40 \% / 60 \%$ strain with an initial SR of $0.6 \Omega / \mathrm{sq}$ at a stretching speed of $60 \mathrm{~mm} / \mathrm{min}$.

stretchability attributed to lower initial SR applied to electrodes comprising of short nanowires as well. At initial SRs of $7.2,3.7$, and $2.0 \Omega / \mathrm{sq}$, the stretchabilities were $60 \%, 70 \%$, and $100 \%$, respectively, while resistance increased at a slower rate. In conclusion, the stretchability is strongly dependent on the initial SR of the AgNWs. Increasing the loading of AgNWs on the PU resulted in electrodes of lower SR, which corresponded to a higher maximum strain and slower rate of increase in resistance [30,41]. This enhanced stretchability might be correlated with an increased number of contacts between nanowires when AgNW density was increased.

The dynamic stability of a stretchable device is of great importance, especially for wearable applications where the movement of the human body is unpredictable. To examine this, AgNW/PU electrodes were stretched to $100 \%$ strain at speeds of $2,4,60,120$, and $480 \mathrm{~mm} / \mathrm{min}$ (all the electrodes had an initial SR of about $0.6 \Omega / \mathrm{sq}$ ). The variation in resistance versus time is shown in Fig. 4(b). For example, when the stretching speed was $60 \mathrm{~mm} / \mathrm{min}$ (strain rate: $8.3 \% / \mathrm{s}$ ), the resistance gradually increased with stretching until reaching the maximum (corresponding to $100 \%$ strain), which was approximately one order of magnitude higher than the initial value. After reaching the maximum, the electrode was released and its resistance gradually decreased. When the strain was completely released, the resistance decreased, resting at 4 times its original value. Similar peak and recovered values were seen for all stretching speeds, from 2 to $480 \mathrm{~mm} / \mathrm{min}$. A dynamically stable strain-resistance relationship was found for our electrodes, over a vast range of stretching speeds, outperforming previously reported results [29]. The obtained dynamic stability might be attributed to the strong adhesion between the AgNWs and the PU. Even at high stretching speeds, the nanowires are firmly adhered to the PU, hence a change in the resistance due to the geometrical change of the AgNW film is dependent only on the applied strain.

Behavior of the fabricated electrodes over multiple stretch-relaxation cycles was investigated. Figure 4(c) 
shows the transient resistance measured during 1,000 cycles, with peak strain at $20 \%$ for electrodes with original SRs of $2.5,1.1$, and $0.6 \Omega /$ sq. For the sample with an original SR of $2.5 \Omega / \mathrm{sq}$, resistance increased from 6 to $18 \Omega$ upon being stretched to $20 \%$ strain, while after relaxation the resistance decreased to around $7 \Omega$, which is a $16.7 \%$ increase on the original value. Figure S5 in the ESM displays the reversible oscillation in resistance over consecutive cycles, which is accompanied by a gradual increase in both peak and baseline resistance. After 1,000 cycles, the baseline and peak resistance had increased to 28 and $40 \Omega$, respectively. However, after the sample had been relaxed for $30 \mathrm{~min}$, the resistance had reduced to $13.7 \Omega$, only twice the original value. This suggests that the gradual increase in resistance was partially due to the viscoelastic properties of the PU. Over 1,000 cycles, all three samples remained highly conductive with peak resistances of only 40.0, 11.1, and $5.9 \Omega$. After the samples had been relaxed for $30 \mathrm{~min}$, their resistances were restored to $13.7,5.1$, and $4.0 \Omega$. Of the three electrodes investigated, those with lower SRs demonstrated better long-term stability, i.e., smaller increases in resistance over time, and smaller oscillations between peak and baseline resistances. Again, increased stretchability can possibly be attributed to an increased amount of interconnections between the nanowires. A similar test was also performed on electrodes with higher initial SRs (transmittance $>80 \%$ ), the results of which can be seen in Fig. S6 in the ESM.

To investigate the maximum strain allowed for multiple stretching-relaxation cycles, electrodes with initial SRs of about $0.6 \Omega / \mathrm{sq}$ were stretched cyclically
1,000 times, with peak strains of $20 \%, 40 \%$, and $60 \%$ (Fig. 4(d)). Higher peak strains leads to a faster increase in resistance. An almost synchronous increase in resistance was seen for peak strains of $40 \%$ and $60 \%$ over the first 200 cycles. However, after this the curves diverged, with the $40 \%$ curve saturating while the $60 \%$ curve continued to increase. After this point, the increase in resistance for the $40 \%$ peak strain sample might be primarily due to the viscoelasticity of the $\mathrm{PU}$, whereas the increase in resistance for the $60 \%$ peak strain sample could be attributed to a combination of the viscoelastic properties of PU and the continuous growth of ruptures within the AgNW network when subjected to a higher strain. Nevertheless, over 1,000 cycles all three samples remain highly conductive, with peak resistances of 66.1, 42.3, and $5.9 \Omega$. After samples had been relaxed for $30 \mathrm{~min}$, the resistances were partially restored to $49.6,13.8$, and $4.0 \Omega$. Even after 1,000 continuous cycles at a peak strain of $60 \%$, the electrode retained a low SR of only $6.5 \Omega / \mathrm{sq}$, which is much lower than other reported cyclic tests $[30,41]$. The electrodes stretched cyclically to $20 \%$ and $40 \%$ recovered fairly well, while the electrode stretched cyclically to $60 \%$ exhibited a more profound permanent increase in resistance.

The length of the AgNWs plays an important role in achieving better mechanical properties. Figure 5(a) shows the comparison between samples with similar transmittances containing short and long nanowires when stretched to $100 \%$ strain. The changes in resistance at $20 \%, 40 \%, 60 \%, 80 \%$, and $100 \%$ strain were $2.7,4.9$, 9.9, 22.0, and 62.0 times for short NWs, and 2.5, 4.3, 7.2, 11.7, and 19.8 times for long NWs, respectively.
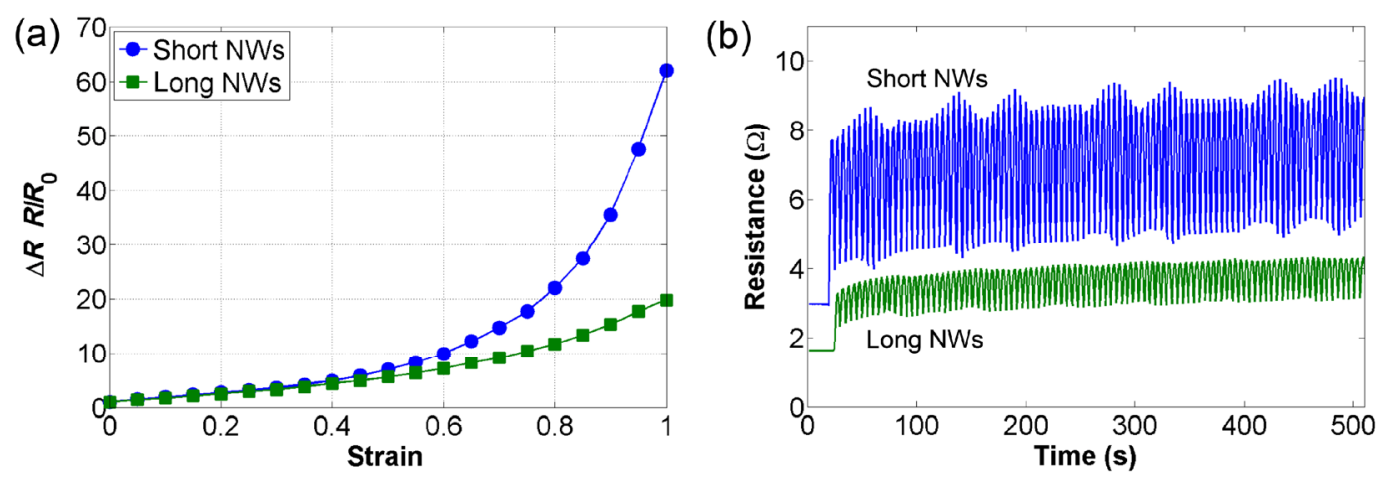

Figure 5 Comparison between short and long NWs. (a) Comparison between short and long NWs with strain up to 100\%. (b) Comparison of transient resistance of short and long NWs measured over 100 cycles of stretching-relaxation at strains of between 0 and $20 \%$. 
The enhancement in stretchability given by using long nanowires is clear, especially at higher strains. As the strain increases, the expansion of the network caused displacement of the nanowires, with the originally interconnected NWs detaching from each other, causing the loss of conductive paths. This phenomenon is naturally faster for shorter NWs than for longer NWs. At small strains, sufficient interconnections between NWs ensure a minimal change in resistance. When the strain was increased to over $40 \%$, enough connections in the long NWs withstood the stretch to minimize the change in resistance. In contrast, most connections in the short wires were detached, leading to a rapid increase in resistance. Another reason for this might be the straightening of NWs on stretching, especially at high strains. The conductive paths are caused by the connection of NWs, number of which in a random network is proportional to NW length. When these NWs have been stretched with a large strain, they tend to change from a curly state to a straight state, diminishing the number of connections between the NWs. As a result, the resistance was increased more significantly in the case of short NWs. Furthermore, even at the same curvatures, longer NWs can withstand larger displacements before they break. The results were further confirmed by the oscillation range of the resistance. Figure 5(b) demonstrates the resistance oscillation for the two kinds of NWs under the same transmittance ( $20 \%$ in this case). The short NWs always show a wide oscillation in comparison with the narrow one seen in the case of long NWs. These results agree with the reasoning mentioned above. An electrode comprising of short NWs retains limited electrical connections which can easily detach or even be destroyed, causing wide resistance oscillation. In contrast, long NWs retain enough connections and display narrow resistance oscillation. These results indicate that the long NWs are strongly favorable for high stretching conditions.

To further understand the cause of increased resistance, we examined an electrode with a transmittance of $20 \%$ by SEM (Fig. 6). The AgNWs showed a uniform distribution network before the stretch test (Fig. 6(a)). However, when the electrode was stretched to $100 \%$ strain, a non-uniform distribution of dense (gray) and sparse (black) clusters of AgNWs was observed (Figs. 6(b) and 6(c)), which corresponded to partially and fully embedded nanowires, respectively. This nonuniform expansion of the nanowire network is probably due to the partial embedding of the network into PU substrate. Upon stretching, the strain applied to the composite cannot be spread uniformly across the partially embedded AgNW network, causing strain localization to occur at its weakest interconnection
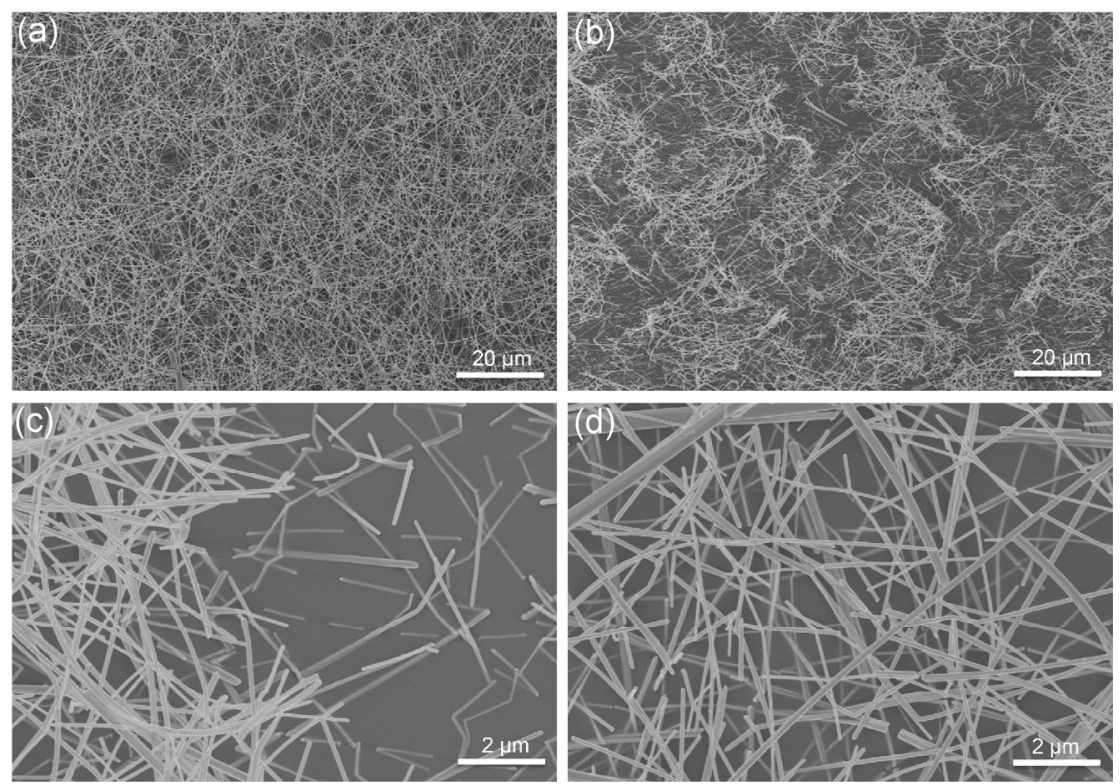

Figure 6 SEM image of a AgNW electrode: (a) in an unstretched state, (b) and (c) under 100\% strain, and (d) in a relaxed state after 1,000 cycles of stretching-relaxation with peak strain of $40 \%$. All samples have initial SRs of $0.6 \Omega / s q$. 
points, rupturing the original network. Buckling or shifting of the partially embedded network might also have happened around fracture areas due to insufficient adhesion to the host material. Figure 6(c) clearly shows the fully embedded material (black) is uniformly stretched, proven by the lower density of nanowires, whereas the partially and un-embedded nanowires (gray) remains dense but with clear break from other parts. This loss of conductive paths due to breaks in the wire-wire interconnections, or in the individual wires themselves (Figs. 6(b) and 6(c)), accounts for the majority of the increase in resistance under stretched conditions. Nevertheless, most conductive paths remain even under $100 \%$ strain after many stretchingrelaxation cycles, maintaining the conductivity of the electrode. Upon release, the partially embedded AgNWs cannot completely follow the recovery of PU. As a consequence, they are unable to return to their initial positions, instead relaxing to the nearby area. Many fractures between nanowires have been observed after 1,000 cycles (Fig. 6(d)), which agrees with the increase in resistance after relaxation mentioned above.
To demonstrate the potential of the HIPL technique and our conductive films for stretchable electronics, we have fabricated two devices based on the present AgNW/PU composite electrodes. The first was a stretchable circuit with a LED chip assembled on AgNW/PU electrodes. A schematic of the device structure and a digital photograph of the fabricated part is shown in Figs. 7(a) and 7(b). High loadings of AgNWs (after HIPL, SR $\approx 6 \Omega /$ sq, and transmittance $\approx$ $68 \%$ ) were used to ensure less variation of resistance during stretching as explained earlier. These were deposited on the top side of the PU to ensure stable operation of the LED. The HIPL-treated AgNW film was then micro-structured by laser ablation to isolate the anode and cathode of the LED, as seen in the optical microscopic image in Fig. 7(c). To match the mechanical properties of the rigid components and the stretchable conductor, an island structure is often used to release the strain on the island in order to protect the rigid components $[3,49,50]$. Here, an island structure made of porous paper was embedded in the PU matrix by a simple thermal pressing step to (a) Top view

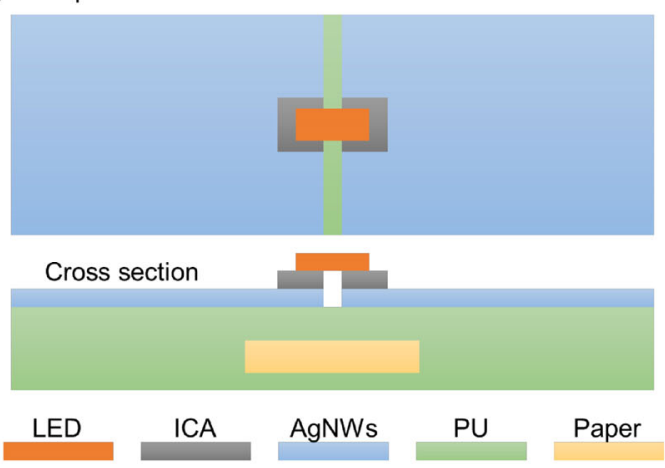

(b)

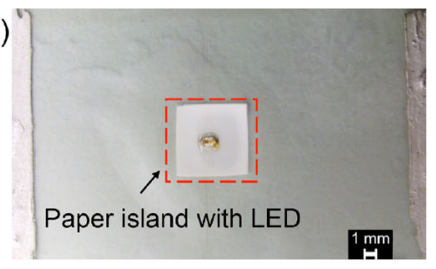

(c)

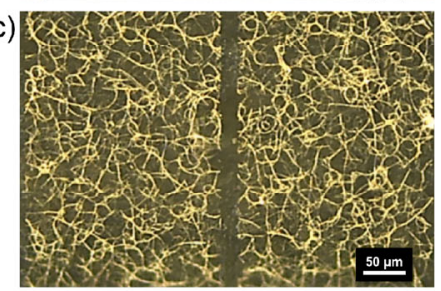

(d)

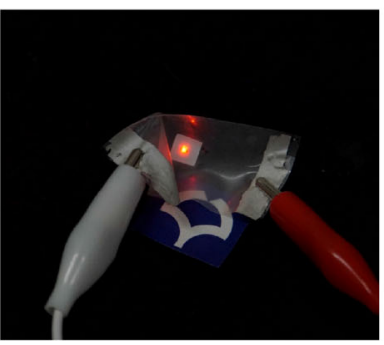

(e)

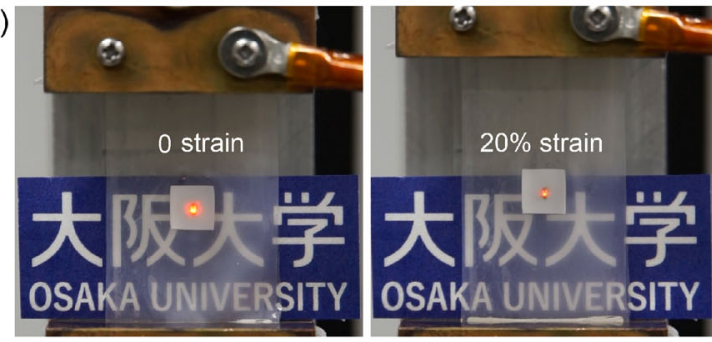

Figure 7 Application of AgNW/PU composite electrodes in semi-transparent and stretchable LEDs. (a) Top-view, and cross-sectional view of the device schematic. (b) Digital photograph of the LED, driven by integrated stretchable conductors. A commercially available LED chip was assembled on the paper island using conductive adhesive. The AgNWs were micro-structured by laser ablation to isolate anodes and cathodes for the LED. (c) Optical microscopic image of the laser ablated AgNWs network. The dark fields indicate the ablated parts. (d) The device is fully resistant to bending or twisting. (e) The device can be cyclically stretched between 0 and $20 \%$ strain. 
act as a flexible carrier for the LED chip. Thanks to the porosity and roughness of the paper, it mechanically interlocked with the surrounding PU layers, forming a strong bond within the structure [51]. The influence of embedding the paper on the strain distribution over the $2 \mathrm{D}$ plane of the resulting device was analyzed by the digital image correlation (DIC) technique. A clear decrease in strain on the island region was observed, corresponding to only $1 \%$ when $20 \%$ strain was applied, or to $10 \%$ when $40 \%$ strain was applied. Further details on the analysis are available in Fig. S7 in the ESM. The resulting device is fully resistant to bending and twisting (Fig. 7(d)), and can be cyclically stretched to $20 \%$ (Fig. 7(e)). Two movies are available in the ESM (Movie S1 and Movie S2) showing the operation of the device under cyclic loading of $20 \%$ strain. These results indicated that the stretchable circuit was perfect for use with existing off-the-shelf rigid electronic components.

The second application explored was a wearable sensor based on the stretchable electrode capable of detecting human motion. A transparent electrode with a transmittance of $78 \%$ was fabricated and attached onto a glove for the detection of finger joint bending. Figure 8 illustrates the behavior of the electrode during the first 10 bending-releasing cycles between $0^{\circ}$ and $90^{\circ}$. When the finger bent, the resistance gradually increased from $\sim 30 \Omega$ to a maximum of $\sim 80 \Omega$. A fully reversible and rather stable change of the resistance between $\sim 30$ and $\sim 80 \Omega$ is clearly visible in the following cycles, corresponding to the bending

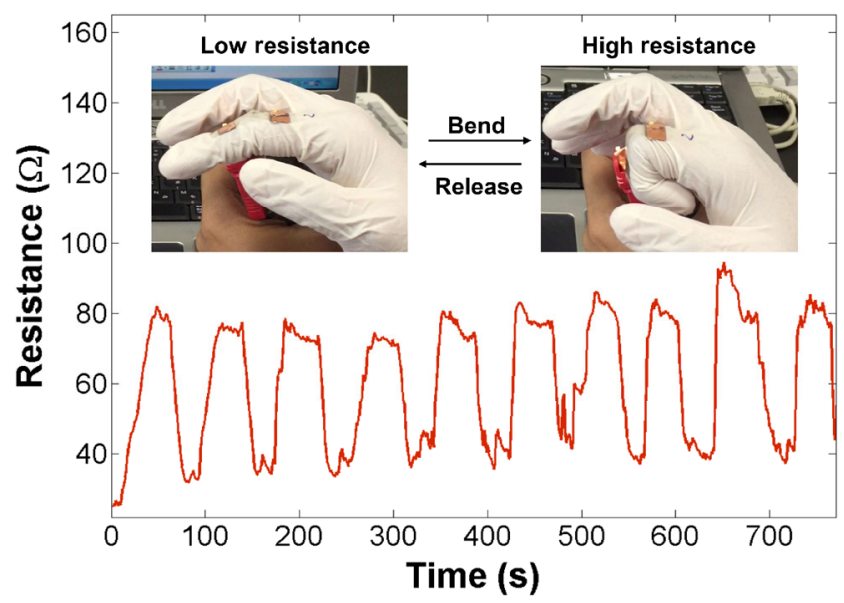

Figure 8 Detection of finger joint motion by transparent (transmittance $=78 \%$ ) electrode. or releasing of the finger. Therefore, the sensor can be employed for accurate motion detection of finger joints and could be used as e-skin for robots. A movie is available in the ESM (Movie S3) showing this process. Furthermore, an electrode of an even higher transmittance of $84 \%$ has been tested likewise, and the results are illustrated in Fig. S8 in the ESM. Similar behavior was confirmed, with an even higher sensitivity (i.e., a larger change in resistance). By reducing the loading of AgNWs, the SR was increased and enhanced sensitivity was obtained due to larger changes in resistance, which were caused by being close to the limit of percolative conduction.

\section{Conclusions}

In summary, a facile fabrication methodology based on HIPL has been proposed and demonstrated for creation of highly conductive and stretchable AgNW/ PU composite electrodes. The ability of HIPL to heat locally instead of globally has resulted in great advantages in control, speed, and energy efficiency in the fabrication process. Strong wire-wire junction conductivity and wire-substrate adhesion were achieved simultaneously with one single step that only takes $50 \mu \mathrm{s}$. The thermal characteristics of the hosting substrate play an important role in this process. Due to the low thermal conductivity of the PU, the extreme heating was contained in the AgNW layer, accelerating the sintering of the nanowires. Additionally, the elevated temperature also resulted in the softening or melting of the PU at interfaces with the AgNWs, which enabled the embedding of the AgNW network into the PU without substantial damage to the whole substrate. The resulting low SR electrode showed excellent stretchability, and an electrode of $0.6 \Omega / \mathrm{sq}$ could survive stretching to $100 \%$ strain without clear deterioration, and retained a SR of only $6.5 \Omega$ /sq even after 1,000 continuous stretching-releasing cycles between 0 and $60 \%$ strain. Moreover, higher AgNW density and longer AgNWs helped to achieve higher allowable strain, slower increases in resistance, and smaller oscillation range of resistance during cyclic stretching-relaxation of the samples. The facile fabrication route overcomes the limitations existing in current fabrication strategies, such as multiple steps, 
time-consuming procedures, and continuous exposure of the sample to high temperatures or pressures that prevent delicate materials from being used. We believe this fabrication method can be scaled up to a future roll-to-roll process and provides a promising strategy for synthesizing next generation stretchable electronic devices. We demonstrated the potential of our technique by applying the fabricated electrode onto a glove as a sensor for finger joint motion, and we fabricated a stretchable LED integrated conductor based on the AgNW/PU composite electrode.

\section{Acknowledgements}

The authors would like to thank the members of Showa Denko K. K. for constructive discussions and encouragement. This work was supported by JSPS Strategic Young Researcher Overseas Visits Program for Accelerating Brain Circulation, the Center of Innovation Program from Japan Science and Technology Agency of JST, JSPS KAKENHI (No. 15K21140), and the Flemish Agency for Innovation by Science and Technology (IWT)-through the program for Strategic Basic Research (SBO) under grant agreement $n^{\circ} 120024$ (Self Sensing Composites).

Electronic Supplementary Material: Supplementary material (simulation of HIPL, further study on the influence of pulsed light irradiation, more results on tensile tests, and movies of the two demonstrative applications) is available in the online version of this article at http://dx.doi.org/10.1007/s12274-015-0921-9.

\section{References}

[1] Rogers, J. A.; Someya, T.; Huang, Y. Materials and mechanics for stretchable electronics. Science 2010, 327, 1603-1607.

[2] Xu, S.; Zhang, Y. H.; Cho, J.; Lee, J.; Huang, X.; Jia, L.; Fan, J. A.; Su, Y. W.; Su, J.; Zhang, H. G. et al. Stretchable batteries with self-similar serpentine interconnects and integrated wireless recharging systems. Nat. Commun. 2013, 4, 1543.

[3] Vanfleteren, J.; Gonzalez, M.; Bossuyt, F.; Hsu, Y.-Y.; Vervust, T.; De Wolf, I.; Jablonski, M. Printed circuit board technology inspired stretchable circuits. MRS Bull. 2012, 37, 254-260.
[4] Vervust, T.; Buyle, G.; Bossuyt, F.; Vanfleteren, J. Integration of stretchable and washable electronic modules for smart textile applications. J. Text. Inst. 2012, 103, 1127-1138.

[5] Bossuyt, F.; Vervust, T.; Vanfleteren, J. Stretchable electronics technology for large area applications: Fabrication and mechanical characterization. IEEE Trans. Comp. Pack. Man. Technol. 2013, 3, 229-235.

[6] Lai, Y.-C.; Huang, Y.-C.; Lin, T.-Y.; Wang, Y.-X.; Chang, C.-Y.; Li, Y.; Lin, T.-Y.; Ye, B.-W.; Hsieh, Y.-P.; Su, W.-F. et al. Stretchable organic memory: Toward learnable and digitized stretchable electronic applications. NPG Asia Mater. 2014, 6, e87.

[7] Feng, X.; Yang, B. D.; Liu, Y. M.; Wang, Y.; Dagdeviren, C.; Liu, Z. J.; Carlson, A.; Li, J. Y.; Huang, Y. G.; Rogers, J. A. Stretchable ferroelectric nanoribbons with wavy configurations on elastomeric substrates. ACS Nano 2011, 5, 3326-3332.

[8] Graz, I. M.; Lacour, S. P. Complementary organic thin film transistor circuits fabricated directly on silicone substrates. Organic Electronics 2010, 11, 1815-1820.

[9] Lipomi, D. J.; Tee, B. C. K.; Vosgueritchian, M.; Bao, Z. Stretchable organic solar cells. Adv. Mater. 2011, 23, 17711775.

[10] Sekitani, T.; Nakajima, H.; Maeda, H.; Fukushima, T.; Aida, T.; Hata, K.; Someya, T. Stretchable active-matrix organic light-emitting diode display using printable elastic conductors. Nat. Mater. 2009, 8, 494-499.

[11] Kaltenbrunner, M.; Sekitani, T.; Reeder, J.; Yokota, T.; Kuribara, K.; Tokuhara, T.; Drack, M.; Schwödiauer, R.; Graz, I.; Bauer-Gogonea, S. et al. An ultra-lightweight design for imperceptible plastic electronics. Nature 2013, 499, 458-463.

[12] Hu, L. B.; Pasta, M.; Mantia, F. L.; Cui, L. F.; Jeong, S.; Deshazer, H. D.; Choi, J. W.; Han, S. M.; Cui, Y. Stretchable, porous, and conductive energy textiles. Nano Lett. 2010, 10, 708-714.

[13] Yamada, T.; Hayamizu, Y.; Yamamoto, Y.; Yomogida, Y.; Izadi-Najafabadi, A.; Futaba, D. N.; Hata, K. A stretchable carbon nanotube strain sensor for human-motion detection. Nat. Nanotechnol. 2011, 6, 296-301.

[14] Niu, Z. Q.; Dong, H. B.; Zhu, B. W.; Li, J. Z.; Hng, H. H.; Zhou, W. Y.; Chen, X. D.; Xie, S. S. Highly stretchable, integrated supercapacitors based on single-walled carbon nanotube films with continuous reticulate architecture. $A d v$. Mater. 2013, 25, 1058-1064.

[15] Yu, Z. B.; Niu, X. F.; Liu, Z. T.; Pei, Q. B. Intrinsically stretchable polymer light-emitting devices using carbon nanotube-polymer composite electrodes. Adv. Mater. 2011, 23, 3989-3994. 
[16] Sekitani, T.; Someya, T. Stretchable, large-area organic electronics. Adv. Mater. 2010, 22, 2228-2246.

[17] Liang, J. J.; Li, L.; Tong, K.; Ren, Z.; Hu, W.; Niu, X. F.; Chen, Y. S.; Pei, Q. B. Silver nanowire percolation network soldered with graphene oxide at room temperature and its application for fully stretchable polymer light-emitting diodes. ACS Nano 2014, 8, 1590-1600.

[18] Xiao, L.; Chen, Z.; Feng, C.; Liu, L.; Bai, Z.-Q.; Wang, Y.; Qian, L.; Zhang, Y. Y.; Li, Q. Q.; Jiang, K. L. et al. Flexible, stretchable, transparent carbon nanotube thin film loudspeakers. Nano Lett. 2008, 8, 4539-4545.

[19] Hu, L. B.; Yuan, W.; Brochu, P.; Gruner, G.; Pei, Q. B. Highly stretchable, conductive, and transparent nanotube thin films. Appl. Phys. Lett. 2009, 94, 161108.

[20] Kim, K. S.; Zhao, Y.; Jang, H.; Lee, S. Y.; Kim, J. M.; Kim, K. S.; Ahn, J.-H.; Kim, P.; Choi, J.-Y.; Hong, B. H. Largescale pattern growth of graphene films for stretchable transparent electrodes. Nature 2009, 457, 706-710.

[21] Chun, K.-Y.; Oh, Y.; Rho, J.; Ahn, J.-H.; Kim, Y.-J.; Choi, H. R.; Baik, S. Highly conductive, printable and stretchable composite films of carbon nanotubes and silver. Nat. Nanotechnol. 2010, 5, 853-857.

[22] Araki, T.; Nogi, M.; Suganuma, K.; Kogure, M.; Kirihara, O. Printable and stretchable conductive wirings comprising silver flakes and elastomers. IEEE Electr. Device Lett. 2011, 32, 1424-1426.

[23] Park, M.; Im, J.; Shin, M.; Min, Y.; Park, J.; Cho, H.; Park, S.; Shim, M.-B.; Jeon, S.; Chung, D.-Y. et al. Highly stretchable electric circuits from a composite material of silver nanoparticles and elastomeric fibres. Nat. Nanotechnol. 2012, 7, 803-809.

[24] Sun, J.-Y.; Zhao, X. H.; Illeperuma, W. R.; Chaudhuri, O.; Oh, K. H.; Mooney, D. J.; Vlassak, J. J.; Suo, Z. G. Highly stretchable and tough hydrogels. Nature 2012, 489, 133-136.

[25] Keplinger, C.; Sun, J.-Y.; Foo, C. C.; Rothemund, P.; Whitesides, G. M.; Suo, Z. G. Stretchable, transparent, ionic conductors. Science 2013, 341, 984-987.

[26] Hu, L. B.; Kim, H. S.; Lee, J.-Y.; Peumans, P.; Cui, Y. Scalable coating and properties of transparent, flexible, silver nanowire electrodes. ACS Nano 2010, 4, 2955-2963.

[27] Xu, F.; Zhu, Y. Highly conductive and stretchable silver nanowire conductors. Adv. Mater. 2012, 24, 5117-5122.

[28] Lee, P.; Lee, J.; Lee, H.; Yeo, J.; Hong, S.; Nam, K. H.; Lee, D.; Lee, S. S.; Ko, S. H. Highly stretchable and highly conductive metal electrode by very long metal nanowire percolation network. Adv. Mater. 2012, 24, 3326-3332.

[29] Hu, W. L.; Niu, X. F.; Zhao, R.; Pei, Q. B. Elastomeric transparent capacitive sensors based on an interpenetrating composite of silver nanowires and polyurethane. Appl. Phys.
Lett. 2013, 102, 083303.

[30] Liang, J. J.; Li, L.; Niu, X. F.; Yu, Z. B.; Pei, Q. B. Elastomeric polymer light-emitting devices and displays. Nat. Photonics 2013, 7, 817-824.

[31] Jiu, J. T.; Nogi, M.; Sugahara, T.; Tokuno, T.; Araki, T.; Komoda, N.; Suganuma, K.; Uchida, H.; Shinozaki, K. Strongly adhesive and flexible transparent silver nanowire conductive films fabricated with a high-intensity pulsed light technique. J. Mater. Chem. 2012, 22, 23561-23567.

[32] Jiu, J. T.; Sugahara, T.; Nogi, M.; Araki, T.; Suganuma, K.; Uchida, H.; Shinozaki, K. High-intensity pulse light sintering of silver nanowire transparent films on polymer substrates: The effect of the thermal properties of substrates on the performance of silver films. Nanoscale 2013, 5, 11820-11828.

[33] Garnett, E. C.; Cai, W. S.; Cha, J. J.; Mahmood, F.; Connor, S. T.; Christoforo, M. G.; Cui, Y.; McGehee, M. D.; Brongersma, M. L. Self-limited plasmonic welding of silver nanowire junctions. Nat. Mater. 2012, 11, 241-249.

[34] Schuller, J. A.; Barnard, E. S.; Cai, W. S.; Jun, Y. C.; White, J. S.; Brongersma, M. L. Plasmonics for extreme light concentration and manipulation. Nat. Mater. 2010, 9, 193-204.

[35] Baffou, G.; Quidant, R.; Girard, C. Heat generation in plasmonic nanostructures: Influence of morphology. Appl. Phys. Lett. 2009, 94, 153109.

[36] Kulkarni, D. D.; Kim, S.; Fedorov, A. G.; Tsukruk, V. V. Light-induced plasmon-assisted phase transformation of carbon on metal nanoparticles. Adv. Funct. Mater. 2012, 22, 2129-2139.

[37] Araki, T.; Sugahara, T.; Jiu, J. T.; Nagao, S.; Nogi, M.; Koga, H.; Uchida, H.; Shinozaki, K.; Suganuma, K. Cu salt ink formulation for printed electronics using photonic sintering. Langmuir 2013, 29, 11192-11197.

[38] Jiu, J.; Araki, T.; Wang, J.; Nogi, M.; Sugahara, T.; Nagao, S.; Koga, H.; Suganuma, K.; Nakazawa, E.; Hara, M. et al. Facile synthesis of very-long silver nanowires for transparent electrodes. J. Mater. Chem. A 2014, 2, 6326-6330.

[39] De, S.; Higgins, T. M.; Lyons, P. E.; Doherty, E. M.; Nirmalraj, P. N.; Blau, W. J.; Boland, J. J.; Coleman, J. N. Silver nanowire networks as flexible, transparent, conducting films: Extremely high DC to optical conductivity ratios. ACS Nano 2009, 3, 1767-1774.

[40] Madaria, A. R.; Kumar, A.; Ishikawa, F. N.; Zhou, C. W. Uniform, highly conductive, and patterned transparent films of a percolating silver nanowire network on rigid and flexible substrates using a dry transfer technique. Nano Res. 2010, 3, 564-573.

[41] Yun, S.; Niu, X. F.; Yu, Z. B.; Hu, W. L.; Brochu, P.; Pei, Q. B. Compliant silver nanowire-polymer composite 
electrodes for bistable large strain actuation. Adv. Mater. 2012, 24, 1321-1327.

[42] Amjadi, M.; Pichitpajongkit, A.; Lee, S.; Ryu, S.; Park, I. Highly stretchable and sensitive strain sensor based on silver nanowire-elastomer nanocomposite. ACS Nano 2014, 8, 5154-5163.

[43] Gaynor, W.; Burkhard, G. F.; McGehee, M. D.; Peumans, P. Smooth nanowire/polymer composite transparent electrodes. Adv. Mater. 2011, 23, 2905-2910.

[44] Akter, T.; Kim, W. S. Reversibly stretchable transparent conductive coatings of spray-deposited silver nanowires. ACS Appl. Mater. Interfaces 2012, 4, 1855-1859.

[45] Zeng, X. Y.; Zhang, Q. K.; Yu, R. M.; Lu, C. Z. A new transparent conductor: Silver nanowire film buried at the surface of a transparent polymer. Adv. Mater. 2010, 22, 4484-4488.

[46] Yu, Z. B.; Zhang, Q. W.; Li, L.; Chen, Q.; Niu, X. F.; Liu, J.; Pei, Q. B. Highly flexible silver nanowire electrodes for shape-memory polymer light-emitting diodes. Adv. Mater.
2011, 23, 664-668.

[47] Hu, W. L.; Niu, X. F.; Li, L.; Yun, S.; Yu, Z. B.; Pei, Q. B. Intrinsically stretchable transparent electrodes based on silvernanowire-crosslinked-polyacrylate composites. Nanotechnology 2012, 23, 344002.

[48] Sun, Y. G.; Gates, B.; Mayers, B.; Xia, Y. N. Crystalline silver nanowires by soft solution processing. Nano Lett. 2002, 2, 165-168.

[49] Hu, X. L.; Krull, P.; de Graff, B.; Dowling, K.; Rogers, J. A.; Arora, W. J. Stretchable inorganic-semiconductor electronic systems. Adv. Mater. 2011, 23, 2933-2936.

[50] Lacour, S. P.; Jones, J.; Wagner, S.; Li, T.; Suo, Z. G. Stretchable interconnects for elastic electronic surfaces. Proc. IEEE 2005, 93, 1459-1467.

[51] Baldan, A. Adhesively-bonded joints and repairs in metallic alloys, polymers and composite materials: Adhesives, adhesion theories and surface pretreatment. J. Mater. Sci. 2004, 39, $1-49$. 